OPEN ACCESS

Edited by:

Robert Jeenchen Chen,

The Ohio State University,

United States

Reviewed by:

Maruti Haranal,

National Heart Institute, Malaysia

Debasis Das,

Narayana Superspeciality

Hospital, India

Michael Hofmann,

University of Zurich, Switzerland

*Correspondence:

Xiaowei Wang

wangxiaowein1@163.com

†These authors have contributed equally to this work and share first

authorship

Specialty section:

This article was submitted to

Heart Surgery,

a section of the journal

Frontiers in Surgery

Received: 27 July 2021 Accepted: 25 August 2021 Published: 17 September 2021

Citation:

Li J, YU D, Song Y, Cheang I and Wang $X$ (2021) Association Between Postoperative Thrombocytopenia and

Outcomes After Coronary Artery

Bypass Grafting Surgery.

Front. Surg. 8:747986.

doi: 10.3389/fsurg.2021.747986

\section{Association Between Postoperative Thrombocytopenia and Outcomes After Coronary Artery Bypass Grafting Surgery}

\author{
Jinghang $\mathrm{Li}^{1+}$, Dongmin $\mathrm{Yu}^{1+}$, Yuanyuan Song ${ }^{1 \dagger}$, lokfai Cheang ${ }^{2}$ and Xiaowei Wang ${ }^{1 \star}$
}

\footnotetext{
'Department of Cardiovascular Surgery, The First Affiliated Hospital of Nanjing Medical University, Nanjing, China,

${ }^{2}$ Department of Cardiology, The First Affiliated Hospital of Nanjing Medical University, Nanjing, China
}

Objectives: The effect of postoperative thrombocytopenia on adverse events among coronary artery bypass graft (CABG) patients remains unclear. This study aims to investigate the association between postoperative thrombocytopenia and perioperative outcomes of CABG.

Methods: This is a retrospective study with MIMIC-III (Medical Information Mart for Intensive Care III) database. Adult patients who underwent CABG were included to analyze the impact of thrombocytopenia in patients' outcomes. Postoperative thrombocytopenia was defined as a platelet count $<100 \times 10^{9} / \mathrm{L}$ on the first day after CABG surgery. A multivariable logistic regression analysis was utilized to adjust the effect of thrombocytopenia on outcomes for baseline and covariates, and to determine the association with outcomes.

Results: A total of 4,915 patients were included, and postoperative thrombocytopenia occurred in 696 (14.2\%) patients. Postoperative thrombocytopenia was not associated with increased 28-day mortality (OR 0.75; 95\% Cl 0.33-1.72; $P=0.496$ ) or in-hospital mortality (OR 0.75; 95\% Cl 0.34-1.63; $P=0.463$ ) after adjusting for confounders. Regarding the secondary outcomes, it was associated with a higher risk of a prolonged stay in the intensive care unit (OR 1.53; 95\% $\mathrm{Cl} 1.18-1.97 ; P=0.001$ ), prolonged hospital stays (OR 1.58; 95\% Cl 1.21-2.06; $P=0.001$ ), prolonged mechanical ventilation time (OR 1.67; 95\% Cl 1.14-2.44; $P=0.009$ ), and a trend toward increased occurrence of massive bleeding (OR 1.41; 95\% Cl 1.00-2.01; $P=0.054)$. There was no significant association between an increased risk of prolonged vasopressor use and the continuous renal replacement therapy rate.

Conclusions: Postoperative thrombocytopenia was associated with prolonged ICU and hospital stays but not with increased perioperative mortality among CABG patients.

Keywords: coronary artery bypass grafting, thrombocytopenia, mortality, MIMIC-III database, perioperation 


\section{INTRODUCTION}

The causes of thrombocytopenia in cardiac surgery are multifactorial and complicated. Studies suggested inflammatory response induced by cardiopulmonary bypass (CPB) as a major factor. The activation of inflammatory cytokines (such as TNF- $\alpha$, IL- 8 , IL-10, IL-6, and IL-1 $\beta)(1,2)$ could induce systemic autoimmune platelet clearance $(3,4)$. The other risk factors included hemodilution, platelet consumption and destruction, heparin-induced thrombocytopenia (HITP), druginduced thrombocytopenia (DITP), etc. (5).

A low platelet count is one common indicator of organ dysfunction in intensive care unit (ICU), which often relates to the severity and mortality of critical diseases (5). Previous studies showed that preoperative thrombocytopenia was associated with both severe bleeding and worse outcomes in both cardiac and non-cardiac surgery $(6,7)$. A recent study of coronary artery bypass grafting (CABG) patients with preoperative thrombocytopenia showed a significant association with severe bleeding and increased 30-day and 1-year mortality (8).

Furthermore, CABG patients receiving antiplatelet therapy exposed to an increased risk of perioperative bleeding and reduced platelet count. As platelet count could reflect a systemic organ function, thrombocytopenia in CABG patients not only relates to bleeding events but also predicts other postoperative adverse events, including acute kidney injury, stroke, and acute myocardial infarction (9-11). Therefore, comprehensively evaluating the impact of perioperative thrombocytopenia in CABG patients is of great importance.

In this study, we characterized the incidence of postoperative thrombocytopenia on the first day after CABG surgery in the MIMIC-III database. Our study aims to explore whether thrombocytopenia in CABG patients has a relationship with an increased risk for postoperative mortality and other adverse events.

\section{METHODS}

\section{Sources of Data}

The current large single-center retrospective cohort study used publicly available data from the Medical Information Mart for Intensive Care (MIMIC) III database conducted by Beth Israel Deaconess Medical Center (BIDMC, Boston, Massachusetts). Study was in accordance with the MIMIC-III guidelines which approved by the institutional review boards. Further details on MIMIC-III ethics are available from its original publication (12). Database contains data from 46,520 ICU patients admitted to the BIDMC from 2001 to 2012, including diagnosis, demographics, procedures, vital signs, laboratory examination results, input and output information, medications, and other clinical variables.

Abbreviations: $\mathrm{CABG}$, coronary artery bypass graft; $\mathrm{BMI}$, body mass index; WBC, white blood cell; APTT, activated partial thromboplastin time; PT, prothrombin time; MAP, mean arterial pressure; SOFA, sequential organ failure assessment; CRRT, continuous renal replacement therapy; ARDS, acute respiratory distress syndrome; AMI, acute myocardial infarction; AKI, acute renal failure; ALI, acute lung injury.

\section{Data Collection and Definitions}

All patients undergoing isolated CABG were enrolled in this analysis. Exclusion criteria were age $<18$ years old; lack of platelet counts record within $24 \mathrm{~h}$ ICU admission, and $>5 \%$ missing variables. The patients' baseline characteristics, comorbidities, vital signs, mean arterial pressure (MAP), laboratory results, use of vasopressors, chest drainage volume, continuous renal replacement therapy (CRRT), mechanical ventilation time, etc. were collected. The sequential organ failure assessment (SOFA) score at admission to the ICU was calculated. Postoperative thrombocytopenia was defined as a platelet count $<100 \times 10^{9} / \mathrm{L}$ on the first day after CABG surgery (13).

The primary endpoints were 28-day mortality from the date of ICU admission and in-hospital mortality. Patient mortality information for discharged patients was gathered from the US Social Security Death Index. The secondary outcomes were length of ICU stay, length of hospital stay, mechanical ventilation time, vasopressor use time, chest drainage volume, and CRRT rate.

\section{Statistical Methods}

Continuous variables with non-normal distributions in the present study are presented as the median \pm interquartile range. Categorical variables are presented as numbers and percentages. Comparison analyses were conducted by using the MannWhitney $U$-test for continuous variables and the $\chi^{2}$ test or Fisher exact test for categorical variables.

Univariable logistic regression analysis was conducted to estimate the relationships between postoperative thrombocytopenia and all outcomes. Then, multivariable logistic regression analysis was performed to adjust for the following covariates: comorbidities (hypertension, congestive heart failure, diabetes, chronic pulmonary, renal failure), age, sex, body mass index (BMI), elective surgery, laboratory tests [white blood cell count (WBC), hemoglobin, lactate, activated partial thromboplastin time (APTT), prothrombin time (PT), creatinine], and vital signs [heart rate, mean arterial pressure, respiratory rate, temperature]. To facilitate logistic regression analysis, all continuous outcome variables were transformed into categorical variables according to the $75 \%$ interquartile range or clinical definition.

Statistical analysis was performed using STATA version 15 (STATA Corp LLC, College Station, TX, USA) and SPSS version 26.0 (IBM, Armonk, NY). $P<0.05$ was considered statistically significant.

\section{RESULTS}

\section{Clinical Characteristics of the Study Population}

A total of 4,915 CABG patients [median age 68.5 (60.576.3) year-old] were included. Median platelet count was 146 $[115-184] \times 10^{9} / \mathrm{L}$. The data selection procedure is presented in Figure 1.

There were 696 (14.2\%) patients had developed postoperative thrombocytopenia. The platelet count was significantly lower in the postoperative thrombocytopenia group (84 
\begin{tabular}{|l|l|l|}
\hline \begin{tabular}{|l|l|} 
Patients underwent CABG surgery \\
from MIMIC-IIIv1.4 \\
$\mathrm{N}=5031$
\end{tabular} & $\begin{array}{l}\text { Exclude patients age }<\text { 18 years older } \\
\mathrm{N}=6\end{array}$ \\
& $\begin{array}{l}\text { Exclude patients without exact platelet record } \\
\text { in 24 hours after ICU admission } \\
\mathrm{N}=67 \\
\mathrm{~N}=4915\end{array}$ \\
Exclude individual with data missing $>5 \%$ \\
$\mathrm{~N}=43$
\end{tabular} \mid

TABLE 1 | Baseline and clinical characteristics of the study population.

\begin{tabular}{|c|c|c|c|}
\hline Variables & $\begin{array}{c}\text { Thrombocytopenia } \\
N=696\end{array}$ & $\begin{array}{l}\text { Non-thrombocytopenia } \\
\qquad N=4,219\end{array}$ & $\boldsymbol{P}$ \\
\hline Age, years & $73.6(65.9,80.0)$ & $67.6(59.6,75.4)$ & $<0.001$ \\
\hline BMl & $26.5(23.6,29.9)$ & $28.2(25.1,31.9)$ & $<0.001$ \\
\hline \multicolumn{4}{|l|}{ Comorbidities } \\
\hline Diabetes, $n$ (\%) & 220 (31.6\%) & 1,667 (39.5\%) & $<0.001$ \\
\hline Chronic pulmonary disease, $n(\%)$ & $88(12.6 \%)$ & $625(14.8 \%)$ & 0.146 \\
\hline Renal failure, $n$ (\%) & 75 (10.8\%) & 419 (9.9\%) & 0.496 \\
\hline \multicolumn{4}{|l|}{ Laboratory tests } \\
\hline WBC, $10^{9} / \mathrm{L}$ & $12.1(9.4,15.0)$ & $14.0(11.1,17.5)$ & $<0.001$ \\
\hline Creatinine, mg/dl & $1.0(0.8,1.2)$ & $0.9(0.8,1.2)$ & 0.012 \\
\hline APTT, seconds & $47.1(37.0,65.2)$ & $37.0(31.6,48.2)$ & $<0.001$ \\
\hline PT, seconds & $16.7(15.5,18.4)$ & $15.2(14.3,16.3)$ & $<0.001$ \\
\hline \multicolumn{4}{|l|}{ Vital signs } \\
\hline Heart rate, bpm & $84.9(79.2,90.0)$ & $84.7(78.9,90.9)$ & 0.966 \\
\hline MAP, mmHg & $74.3(70.6,78.2)$ & $74.2(70.6,78.4)$ & 0.633 \\
\hline Respiratory rate, bpm & $16.1(14.6,18.1)$ & $16.9(15.3,18.8)$ & $<0.001$ \\
\hline Temperature, ${ }^{\circ} \mathrm{C}$ & $37.7(37.3,38.1)$ & $37.6(37.2,38.1)$ & 0.04 \\
\hline Elective surgery, $n(\%)$ & $263(37.8 \%)$ & $1,509(35.8 \%)$ & 0.307 \\
\hline SOFA score & $6(5,8)$ & $4(3,6)$ & $<0.001$ \\
\hline SOFA score>2, $n$ (\%) & 685 (98.4\%) & 3,298 (78.2\%) & $<0.001$ \\
\hline
\end{tabular}

Values are expressed as median (interquartile range), or $n$ (\%).

BMI, body mass index; WBC, white blood cell; APTT, activated partial thromboplastin time; PT, prothrombin time; MAP, mean arterial pressure; SOFA, sequential organ failure assessment. In bold are statistically significant values.

[71-92] vs. $\left.155[128-191] \times 10^{9} / \mathrm{L}, P<0.001\right)$. The baseline characteristics between groups with and without postoperative thrombocytopenia are presented in Table 1. The postoperative thrombocytopenia group was older (median age 73.6 vs. 67.6 years old); had fewer males (68.5 vs. $75.4 \%$ ); had a lower BMI, $\mathrm{WBC}$, hemoglobin level, respiratory rate, and diabetes rate; and had a higher congestive heart failure rate, lactate level, APTT, PT and SOFA score. There were no significant differences in heart rate, MAP, comorbidity of hypertension, chronic pulmonary disease, renal failure, and elective surgery between groups.

\section{Related Risk Factors for Postoperative Thrombocytopenia}

The multivariable logistic regression analysis showed that age (OR 1.042; 95\% CI 1.028-1.055), lactate (OR 1.221; 95\% CI 1.143-1.306), APTT (OR 1.006; 95\% CI 1.001-1.011), and 
TABLE 2 | The risk factors of postoperative thrombocytopenia in multivariable logistic regression analysis.

\begin{tabular}{lccc}
\hline Variables & OR & $\mathbf{9 5 \%} \mathbf{C l}$ & P-value \\
\hline Age & 1.042 & $1.028,1.055$ & $<\mathbf{0 . 0 0 1}$ \\
BMl & 0.963 & $0.939,0.988$ & $\mathbf{0 . 0 0 4}$ \\
WBC & 0.892 & $0.866,0.918$ & $<\mathbf{0 . 0 0 1}$ \\
Hemoglobin & 0.831 & $0.759,0.910$ & $<\mathbf{0 . 0 0 1}$ \\
Lactate & 1.221 & $1.143,1.306$ & $<\mathbf{0 . 0 0 1}$ \\
APTT & 1.006 & $1.001,1.011$ & $\mathbf{0 . 0 1 1}$ \\
PT & 1.083 & $1.041,1.126$ & $<\mathbf{0 . 0 0 1}$
\end{tabular}

Estimates are odds ratios with $95 \%$ confidence interval.

$B M I$, body mass index; WBC, white blood cell; APTT, activated partial thromboplastin time; PT, prothrombin time.

In bold are statistically significant values.

PT (OR 1.083; 95\% CI 1.041-1.126) were associated with a significantly higher risk of postoperative thrombocytopenia; BMI (OR 0.963; 95\% CI 0.939-0.988), WBC (OR 0.892; 95\% CI 0.866-0.918), and hemoglobin (OR 0.831; 95\% CI 0.759-0.910) were associated with a significantly lower risk of postoperative thrombocytopenia (Table 2).

\section{Impact of Postoperative Thrombocytopenia on Primary and Secondary Outcomes}

Univariate logistic regression analysis showed both the primary outcomes, 28 -day mortality (3.0 vs. $1.2 \%, P=0.001)$ and inhospital mortality (3.4 vs. $1.4 \%, P=0.001$ ), were significantly increased in postoperative thrombocytopenia patients. While the secondary outcomes, length of ICU stay $(2.8[1.3,4.6]$ vs. $2.2[1.2$, $3.9], P<0.001)$, length of hospital stay $(8.3[6.2,12.7]$ vs. $7.6[5.4$, 11.0], $P<0.001)$, length of ventilation time $(12.0[4.7,21.7]$ vs. $5.9[3.5,15.6], P<0.001)$, length of vasopressor usage (14.2 [3.0, $38.4]$ vs. $12.0[1.3,29.9], P<0.001)$, and chest drainage volume $(1,062.5[762.5,1,648.8]$ vs. $800[535,1,150], P<0.001)$, were also significantly increased in postoperative thrombocytopenia patients (Table 3).

Further multivariate logistic regression analysis showed that 28 -day mortality (OR 0.75 ; 95\% CI $0.33-1.72 ; P=0.495$ ) and inhospital mortality (OR 0.75 ; $95 \%$ CI $0.34-1.63 ; P=0.463$ ) did not show significance increased risk in patients with postoperative thrombocytopenia after adjusting with the confounders. For the secondary outcomes, postoperative thrombocytopenia was associated with a higher risk of an ICU stay $\geq 3$ days (OR 1.53; $95 \%$ CI 1.18-1.97; $P=0.001$ ), hospital stay $\geq 10$ days (OR 1.58 ; 95\% CI 1.21-2.06; $P=0.001)$, mechanical ventilation time $\geq 48 \mathrm{~h}$ (OR 1.67; 95\% CI 1.14-2.44; $P=0.009$ ), and a trend toward an increasing risk with chest drainage volume $\geq 1,000 \mathrm{ml}$ (OR 1.41; 95\% CI 1.00-2.01; $P=0.054$ ) (Table 4).

\section{DISCUSSION}

In this retrospective study, postoperative thrombocytopenia $(14.2 \%)$ often occurred in the first day after CABG surgery among of 4,915 patients. The primary outcome -28-day mortality and in-hospital mortality were significantly higher in the patients with postoperative thrombocytopenia. In further multivariable logistic regression analysis, although postoperative thrombocytopenia had a significantly increased risk of prolonged ICU stay, prolonged hospital stays, prolonged mechanical ventilation time, increased risk of massive bleeding, results did not find significant difference after adjusting with cofounders in the primary outcome.

Platelets play an important role in the pathogenesis of multiple organ dysfunction, including acute respiratory distress syndrome (ARDS), acute myocardial infarction (AMI), stroke, massive bleeding, and acute kidney injury (AKI), major bleeding, and could act as a powerful prognostic indicator in critical patients $(8,10,14-16)$. Therefore, hypothesis suggested that decreased platelet counts might also associated with an inflammatory end-organ injury and coagulation disorders in cardiac surgery.

However, the role of thrombocytopenia especially in postoperative CABG patients remained controversial. A retrospective study of 4,217 adult CABG surgery patients showed that postoperative thrombocytopenia (platelet count $\leq 74 \times 10^{9} / \mathrm{L}$ ) was independently associated with an increased risk of 30-day mortality (10). Study of 7,189 CABG surgery patients, preoperative thrombocytopenia (platelet count $\leq 150$ $\times 10^{9} / \mathrm{L}$ ) was also associated with an increased risk of both in-hospital/30-day mortality and 1-year mortality (8). On the contrary, the ASCERT study, which included 348,341 CABG patients, did not find platelet count as a predictor of both short-term and long-term mortality after CABG surgery (17). Our results are similar to ASCERT study, thrombocytopenia after CABG surgery did not show significance in the increased risk of short-term mortality.

Platelet as the key component in the process of hemostasis and thrombosis, thrombocytopenia is an important risk factor for postoperative bleeding in most studies of cardiac and non-cardiac surgery (18). Study reported that reduced platelet count was associated with increased postoperative bleeding after cardiac surgery (19); while prospective study of 323 cardiac surgery patients also demonstrated an association of thrombocytopenia with excessive bleeding, and that preoperative platelet count was an independent risk factor for excessive bleeding after cardiac surgery (20). Therefore, the thrombocytopenia cohort had a higher pericardial/chest drainage volume and a lower hemoglobin level, which consisted in our multivariate model. However, platelet count only reflects partial function of the platelet itself and the coagulation function (21). While thrombocytopenia does associate with the risk of adverse outcomes, proper perioperative management might significantly decrease the impact in mortality, especially for CABG patients which usually need to receive anti-platelet management (22).

In the inflammatory aspects, cardiac surgery has a higher risk of acute lung injury (ALI) and/or acute respiratory distress syndrome (ARDS) than non-cardiac surgery and often needs longer ventilation support. The inflammatory response associated with cardiac surgery, blood transfusion and microthrombogenesis may contribute to ALI or ARDS after cardiac surgery. Previous studies have revealed that platelets also 
TABLE 3 | Clinical outcomes between study cohorts.

\begin{tabular}{|c|c|c|c|}
\hline Outcomes & $\begin{array}{l}\text { Thrombocytopenia } \\
\qquad N=696\end{array}$ & $\begin{array}{l}\text { Non-thrombocytopenia } \\
\qquad N=4,219\end{array}$ & $P$-value \\
\hline \multicolumn{4}{|l|}{ Primary outcomes } \\
\hline 28-day mortality, $n$ (\%) & $21(3.0 \%)$ & $50(1.2 \%)$ & 0.001 \\
\hline In-hospital mortality, n (\%) & $24(3.4 \%)$ & $61(1.4 \%)$ & 0.001 \\
\hline \multicolumn{4}{|l|}{ Secondary outcomes } \\
\hline Length of ICU stay, days & $2.8(1.3,4.6)$ & $2.2(1.2,3.9)$ & $<0.001$ \\
\hline Length of hospital stay, days & $8.3(6.2,12.7)$ & $7.6(5.4,11.0)$ & $<0.001$ \\
\hline Length of ventilation time, hours & $12.0(4.7,21.7)$ & $5.9(3.5,15.6)$ & $<0.001$ \\
\hline Length of vasopressor time, hours & $14.2(3.0,38.4)$ & $12.0(1.3,29.9)$ & $<0.001$ \\
\hline Pericardial and chest drainage, $\mathrm{ml}$ & $1,062(762,1,648)$ & $800(535,1,150)$ & $<0.001$ \\
\hline CRRT first day & $11(1.6 \%)$ & $62(1.5 \%)$ & 0.738 \\
\hline
\end{tabular}

Values are expressed as median (interquartile range), or $n$ (\%).

CRRT, continuous renal replacement therapy.

In bold are statistically significant values.

TABLE 4 | The associations between postoperative thrombocytopenia and clinical outcomes.

\begin{tabular}{|c|c|c|c|c|}
\hline & \multicolumn{2}{|c|}{ Univariate analysis } & \multicolumn{2}{|c|}{ Multivariate analysis } \\
\hline 28-day mortality & $2.59(1.55,4.35)$ & $<0.001$ & $0.75(0.33,1.72)$ & 0.496 \\
\hline In-hospital mortality & $2.43(1.51,3.93)$ & $<0.001$ & $0.75(0.34,1.63)$ & 0.463 \\
\hline \multicolumn{5}{|l|}{ Secondary outcomes } \\
\hline Length of hospital stay $\geq 10$ days & $1.35(1.15,1.60)$ & $<0.001$ & $1.58(1.21,2.06)$ & 0.001 \\
\hline Length of ventilation time $\geq 48 \mathrm{~h}$ & $1.99(1.57,2.52)$ & $<0.001$ & $1.67(1.14,2.44)$ & 0.009 \\
\hline Length of vasopressor time $\geq 24 \mathrm{~h}$ & $1.23(1.04,1.46)$ & 0.018 & $1.11(0.84,1.45)$ & 0.464 \\
\hline Pericardial and chest drainage $\geq 1,000 \mathrm{ml}$ & $2.39(1.75,3.26)$ & $<0.001$ & $1.41(1.00,2.01)$ & 0.054 \\
\hline CRRT first day & $1.08(0.56,2.06)$ & 0.823 & $1.13(0.23,3.82)$ & 0.846 \\
\hline
\end{tabular}

Estimates are odds ratios with $95 \%$ confidence interval.

CRRT, continuous renal replacement therapy.

In bold are statistically significant values.

contribute to the pathophysiological process of ALI (23). Platelets play a critical role in protecting the integrity of the basal alveolarcapillary barrier and regulating alveolar capillary permeability. When platelet counts are severely decreased, the function of the endothelial barrier is often impaired, leading to increased microvessel permeability, alveolar neutrophil infiltration, and lung injury (24). In the present study, the CABG patients with thrombocytopenia had a significantly prolonged mechanical ventilation time (12 vs. $5.9 \mathrm{~h}$ ) and a 1.67 -fold risk of mechanical ventilation time $\geq 48 \mathrm{~h}$ in the multivariate analysis.

Since postoperative thrombocytopenia count may be caused by inflammation associated with $\mathrm{CPB}$, hemodilution, platelet consumption and destruction, the decrease in postoperative platelet contributes to a systemic adverse mechanism and may exert negative effects by increasing the risk of bleeding, blood transfusion, ALI, poor organ perfusion, and systemic inflammatory response. Although the direct mechanism of postoperative thrombocytopenia on these adverse events has not been well-studied. Our results provided additional evidence regarding platelet in the prognosis of CABG patients, and also established a relationship between thrombocytopenia and other major adverse events. The findings of our study add to the accumulating evidence that postoperative thrombocytopenia is an important risk factor in CABG patients.

\section{LIMITATIONS}

This study has several limitations. First, this was a retrospective study based on a public database of the MIMIC III, several potential risk factors and covariates were not available for analysis. For example, the perioperative antiplatelet drug use, types of CABG procedures (off-pump/on-pump), perioperative blood transfusion, etc. are lack in our cohort, which might generate certain bias in our results. Second, this is a singlecenter study, and the quality of perioperative management and surgical practices may differ from other institutions. Finally, we 
only studied the impact of the platelet count on CABG surgery, and the influence of platelet function was not considered in this study. Platelet function abnormalities may be a more powerful influencing factor for advent events. Studies with multi-center large cohort, integral covariates analysis, and platelet function are needed to further explore the effects and mechanisms.

\section{CONCLUSION}

The results of this retrospective study showed that CABG patients with postoperative thrombocytopenia had a significantly increased risk of a prolonged ICU stay, prolonged hospital stay, prolonged mechanical ventilation time and massive bleeding, but the risk of 28-day and in-hospital death were not significantly increased. Further studies using data from other medical centers will be needed to provide further validation of these findings, and the potential mechanism of postoperative thrombocytopenia and its influence on adverse outcomes should be investigated.

\section{DATA AVAILABILITY STATEMENT}

The original contributions presented in the study are included in the article/supplementary material, further inquiries can be directed to the corresponding author/s.

\section{REFERENCES}

1. McBride WT, Armstrong MA, Crockard AD, McMurray TJ, Rea JM. Cytokine balance and immunosuppressive changes at cardiac surgery: contrasting response between patients and isolated CPB circuits. Br J Anaesth. (1995) 75:724-33. doi: 10.1093/bja/75.6.724

2. Kawamura T, Wakusawa R, Okada K, Inada S. Elevation of cytokines during open heart surgery with cardiopulmonary bypass: participation of interleukin 8 and 6 in reperfusion injury. Can J Anaesth = Journal canadien d'anesthesie. (1993) 40:1016-21. doi: 10.1007/BF03009470

3. Hirai S. Systemic inflammatory response syndrome after cardiac surgery under cardiopulmonary bypass. Ann Thorac Cardiovasc Surg. (2003) 9:365-70.

4. Laffey JG, Boylan JF, Cheng DC. The systemic inflammatory response to cardiac surgery: implications for the anesthesiologist. Anesthesiology. (2002) 97:215-52. doi: 10.1097/00000542-20020700000030

5. Zarychanski R, Houston DS. Assessing thrombocytopenia in the intensive care unit: the past, present, and future. Hematol Am Soc Hematol Educ Program. (2017) 2017:660-6. doi: 10.1182/asheducation-2017.1.660

6. Glance LG, Blumberg N, Eaton MP, Lustik SJ, Osler TM, Wissler R, et al. Preoperative thrombocytopenia and postoperative outcomes after noncardiac surgery. Anesthesiology. (2014) 120:62-75. doi: 10.1097/ALN.0b013e3182a4441f

7. Glyn WD, Susan BL, Chandra R. Factors associated with blood loss and blood product transfusions: a multivariate analysis in children after open-heart surgery. Anesth Anal. (1999) 89:57-64. doi: 10.1097/00000539-199907000-00011

8. Nammas W, Dalén M, Rosato S, Gherli R, Reichart D, Gatti G, et al. Impact of preoperative thrombocytopenia on the outcome after coronary artery bypass grafting. Platelets. (2019) 30:480-6. doi: 10.1080/09537104.2018. 1466389

9. Ranucci M. Hemostatic and thrombotic issues in cardiac surgery. Semin Thromb Hemost. (2015) 41:84-90. doi: 10.1055/s-0034-1398383

10. Kertai MD, Zhou S, Karhausen JA, Cooter M, Jooste E, Li YJ, et al. Platelet counts, acute kidney injury, and mortality after

\section{ETHICS STATEMENT}

Ethical review and approval was not required for the study on human participants in accordance with the local legislation and institutional requirements. Written informed consent for participation was not required for this study in accordance with the national legislation and the institutional requirements.

\section{AUTHOR CONTRIBUTIONS}

JL designed the study and coordinated the study. XW provided administrative support. DY and YS gave provision of study materials or patients. JL and IC collected and assembled the data. IC analyzed and interpreted the data. All authors were participated in the manuscript writing and read and approved the final manuscript.

\section{ACKNOWLEDGMENTS}

We appreciate the assistance of Dr. Yanxiu Li and Dr. Zhongwen Zhang in supporting the statistical analysis. We would also like to thank the initial administration support of Dr. Xiangrong Zuo and Prof. Quan Cao. coronary artery bypass grafting surgery. Anesthesiology. (2016) 124:339-52. doi: 10.1097/ALN.0000000000000959

11. Karhausen JA, Smeltz AM, Akushevich I, Cooter M, Podgoreanu MV, Stafford-Smith M, et al. Platelet counts and postoperative stroke after coronary artery bypass grafting surgery. Anesth Analg. (2017) 125:1129-39. doi: 10.1213/ANE.0000000000002187

12. Johnson AEW, Pollard TJ, Shen L, Lehman LWH, Feng M, Ghassemi M, et al. MIMIC-III, a freely accessible critical care database. entific Data.

13. Thiolliere F, Serre-Sapin AF, Reignier J, Benedit M, Constantin JM, Lebert C, et al. Epidemiology and outcome of thrombocytopenic patients in the intensive care unit: results of a prospective multicenter study. Intensive Care Med. (2013) 39:1460-8. doi: 10.1007/s00134-0132963-3

14. Hui P, Cook DJ, Lim W, Fraser GA, Arnold DM. The frequency and clinical significance of thrombocytopenia complicating critical illness: a systematic review. Chest. (2011) 139:271-8. doi: 10.1378/chest.10-2243

15. Williamson DR, Lesur O, Tétrault JP, Nault V, Pilon D. Thrombocytopenia in the critically ill: prevalence, incidence, risk factors, and clinical outcomes. Can J Anaesth = Journal canadien dianesthesie. (2013) 60:64151. doi: 10.1007/s12630-013-9933-7

16. Raith EP, Udy AA, Bailey M, McGloughlin S, MacIsaac C, Bellomo $\mathrm{R}$, et al. Prognostic Accuracy of the SOFA Score, SIRS Criteria, and qSOFA Score for In-Hospital Mortality Among Adults With Suspected Infection Admitted to the Intensive Care Unit. JAMA. (2017) 317:290300. doi: 10.1001/jama.2016.20328

17. Shahian DM, O'Brien SM, Sheng S, Grover FL, Mayer JE, Jacobs JP, et al. Predictors of long-term survival after coronary artery bypass grafting surgery: results from the Society of Thoracic Surgeons Adult Cardiac Surgery Database (the ASCERT study). Circulation. (2012) 125:1491500. doi: 10.1161/CIRCULATIONAHA.111.066902

18. Weil IA, Kumar P, Seicean S, Neuhauser D, Seicean A. Platelet count abnormalities and peri-operative outcomes in adults undergoing elective, non-cardiac surgery. PLoS ONE. (2019) 14:e0212191. doi: 10.1371/journal.pone.0212191

19. Ranucci M. The interaction between preoperative platelet count and function and its relationship with postoperative bleeding in cardiac 
surgery. Platelets. (2017) 28:794-8. doi: 10.1080/09537104.2017. 1280148

20. Lopes CT, Brunori EF, Cavalcante AM, Moorhead SA, Swanson E, Lopes Jde L, et al. Factors associated with excessive bleeding after cardiac surgery: a prospective cohort study. Heart Lung: J Crit Care. (2016) 45:64-9. e2. doi: 10.1016/j.hrtlng.2015.09.003

21. Grommes J, Alard JE, Drechsler M, Wantha S, Mörgelin M, Kuebler WM, Jacobs M, et al. Disruption of platelet-derived chemokine heteromers prevents neutrophil extravasation in acute lung injury. Am J Respir Crit Care Med. (2012) 185:628-36. doi: 10.1164/rccm.201108-1533OC

22. Agarwal S. Platelet function testing in cardiac surgery. Transfus Med. (2016) 26:319-29. doi: 10.1111/tme.12335

23. Ho-Tin-Noé B, Demers M, Wagner DD. How platelets safeguard vascular integrity. J Thromb Haemost. (2011) 9 (Suppl 1):5665. doi: 10.1111/j.1538-7836.2011.04317.x

24. Karzai W, Priebe HJ. Aspirin and mortality from coronary bypass surgery. $N$ Engl J Med. (2003) 348:1057-9; author reply-9. doi: 10.1056/NEJM200303133481114
Conflict of Interest: The authors declare that the research was conducted in the absence of any commercial or financial relationships that could be construed as a potential conflict of interest.

Publisher's Note: All claims expressed in this article are solely those of the authors and do not necessarily represent those of their affiliated organizations, or those of the publisher, the editors and the reviewers. Any product that may be evaluated in this article, or claim that may be made by its manufacturer, is not guaranteed or endorsed by the publisher.

Copyright (c) $2021 \mathrm{Li}, \mathrm{Yu}$, Song, Cheang and Wang. This is an open-access article distributed under the terms of the Creative Commons Attribution License (CC BY). The use, distribution or reproduction in other forums is permitted, provided the original author(s) and the copyright owner(s) are credited and that the original publication in this journal is cited, in accordance with accepted academic practice. No use, distribution or reproduction is permitted which does not comply with these terms. 